\title{
The impact of patellar resurfacing in two-stage revision of the infected total knee arthroplasty.
}

\author{
Aaron Glynn \\ Rothman Institute at Thomas Jefferson University \\ Ronald Huang \\ Rothman Institute at Thomas Jefferson University \\ Javad Mortazavi \\ Rothman Institute at Thomas Jefferson University \\ Javad Parvizi MD \\ Rothman Institute, Thomas Jefferson University
}

Follow this and additional works at: https://jdc.jefferson.edu/rothman_institute

Part of the Surgery Commons

Let us know how access to this document benefits you

\section{Recommended Citation}

Glynn, Aaron; Huang, Ronald; Mortazavi, Javad; and Parvizi, Javad MD, "The impact of patellar resurfacing in two-stage revision of the infected total knee arthroplasty." (2014). Rothman Institute Faculty Papers. Paper 48.

https://jdc.jefferson.edu/rothman_institute/48

This Article is brought to you for free and open access by the Jefferson Digital Commons. The Jefferson Digital Commons is a service of Thomas Jefferson University's Center for Teaching and Learning (CTL). The Commons is a showcase for Jefferson books and journals, peer-reviewed scholarly publications, unique historical collections from the University archives, and teaching tools. The Jefferson Digital Commons allows researchers and interested readers anywhere in the world to learn about and keep up to date with Jefferson scholarship. This article has been accepted for inclusion in Rothman Institute Faculty Papers by an authorized administrator of the Jefferson Digital Commons. For more information, please contact: JeffersonDigitalCommons@jefferson.edu. 
As submitted to:

The Journal of arthroplasty

And later published as:

The Impact of Patellar Resurfacing in Two-Stage Revision of the Infected Total Knee Arthroplasty

Volume 29, Issue 7, pages: 1439-1442, July 2014

DOI: 10.1016/j.arth.2013.07.018

\author{
Aaron Glynn MD \\ Ronald Huang MD \\ Javad Mortazavi MD \\ Javad Parvizi MD, FRCS
}

The Rothman Institute at Thomas Jefferson University, Philadelphia, PA 


\begin{abstract}
:
Evidence for optimal management of the patellofemoral joint in revision surgery for the infected TKA is limited. We reviewed 69 infected TKAs undergoing two-stage revision. Fifty four patellae were resurfaced, 11 had patelloplasty performed, two were augmented with trabecular metal, one had impaction grafting, and one knee underwent patellectomy. Average follow-up was 4.5 years. The patients that received patellar resurfacing at re-implantation experienced statistically significant improvements in KSS pain score, functional KSS, and patellar score (P b 0.03). One further patient treated with impaction grafting improved significantly in terms of pain and function. Patients treated with patelloplasty, trabecular metal augmentation, or patellectomy did not have significant improvements in clinical or functional outcome. Patient age, use of dynamic vs. static spacer, use of extensor mechanism release, and differences in Charlson index did not seem to statistically affect outcome. We recommend that every effort should be made to minimize patellar bone loss in first stage resection, as inability to resurface the patella at time of reimplantation may adversely affect patient outcome.
\end{abstract}




\section{Introduction:}

Periprosthetic joint infection (PJI) is a devastating outcome following total knee arthroplasty (TKA), occurring in approximately $2 \%$ of cases [1,2]. Two-stage revision is currently the most reliable treatment option for an infected TKA [2]. This consists of complete resection of components, insertion of an antibiotic-loaded spacer block, and delayed reimplantation.

Revision of the infected TKA poses unique surgical challenges for the patella: First, it may be necessary to remove any well-fixed patellar components, potentially risking patellar bone loss. Second, two-stage revisions require the placement of an antibiotic-loaded cement spacer; this inhibits knee flexion, promotes quadriceps and collateral ligament contracture, and can occasionally migrate and erode the patella [3]. Third, surgical exposure must be expanded in TKA revisions in order to view the entire knee joint, and it may necessary to release the extensor mechanism. This can be done through a quadriceps snip, described by Insall, or a V-Y quadricepsplasty when scar contraction of the quadriceps is encountered $[4,5]$. Osteotomy of the tibial tubercle may be helpful in patients with multiple irrigation and debridements since repeated V-Y incisions may cause excessive scarring [6]. Any of these methods can result in post-operative extensor mechanism dysfunction.

Treatment options for the patella at reimplantation include a) resurfacing with a new prosthesis where this is possibe, b) leaving the patella unresurfaced, but debriding osteophytes and necrotic bone (patelloplasty), c) bone grafting, d) implantation of a tantalum porous augment with resurfacing, or e) patellectomy. The purposes of this study are (1) to assess whether patellar resurfacing results in improved outcome compared to other treatment options (2) to assess 
whether spacer type or extensor mechanism release affect functional outcomes following two stage revision of the infected TKA.

\section{Materials and Methods:}

Following institutional review board approval, we retrospectively identified 204 patients that underwent two stage exchange arthroplasty for treatment of an infected total knee arthroplasty at our institution between February 1997 and June 2010. Patients were excluded from our study if they (1) had a pre-established extensor mechanism problem before resection arthroplasty (7 patients), (2) had a documented knee revision surgery involving the patella between the primary procedure and resection stage (66 patients), (3) had repeat revision following the two-stage exchange (19 patients), (4) had a lower limb amputation following twostage exchange (one patient), or (5) had less than one year of follow-up (42 patients). The remaining 69 patients comprised our study cohort.

Our study group comprised 39 males (39 knees) and 30 females (30 knees). The average age was 68.7 years (range, 45-89) at the time of resection. The average mean body mass index (BMI) was $33.2 \mathrm{~kg} / \mathrm{m} 2$ (range, $18.8-63.0 \mathrm{~kg} / \mathrm{m} 2$ ). Patient co-morbidities were also collected and graded according to the Charlson Co-morbidity Index [7].

Irrigation and debridement was elected for 14 knees (13\%) as the initial treatment for infection. These patients eventually underwent two-stage revision arthroplasty due to recurrent infection. The remaining 55 knees underwent resection arthroplasty followed by delayed reimplantation as the initial treatment for infection. Prior incisions were utilized and medial parapatellar arthrotomy performed for access to the knee joint. For all patients, tissue specimens were collected intraoperatively for Gram stain and culture. 
During resection, all hardware and cement was removed, including the patella button where present, and soft tissue debridement was performed on bony surfaces. A patellar button was not present in 5 knees during the resection arthroplasty. At reimplantation, the patella was resurfaced wherever feasible. The same three criteria as outlined by Della Valle et al were used to determine patellar suitability for resurfacing, namely: Bone thickness of at least $10-12 \mathrm{~mm}$, an intact patella without fracture, and adequate vascularization [6]. Patelloplasty comprised shaving of the retained patella with removal of osteophytes.

Fifty four patellae underwent resurfacing, eleven knees had patelloplasty performed, two knees had trabecular metal augments sewn to the patella, one had impaction grafting, and one knee underwent patellectomy. Clinical and functional outcomes of these patients were evaluated using the functional Knee Society Score (KSS), KSS pain score, [8] and a patellar score as described by Feller [9]. This latter allocates 15 points for anterior knee pain and 5 points for each of quadriceps strength, ability to rise from a chair and stairclimbing. It is scored from 3 (worst) to 30 (best outcome). A patellar score was included to address the limitations of the KSS in showing differences in pain and function relating specifically to the patellofemoral joint. Questionnaires were obtained at follow-up visits in the office and administered to patients via telephone. Average duration of follow-up was 4.5 years (range: 1.0-10.9 years).

Further data on specifics of spacer usage and surgical exposure during re-implantation were collected from patient charts. Fifty two knees received static spacers and seventeen received dynamic spacers. Three knees $(4.3 \%)$ required a quadriceps snip, four knees (5.8\%) required V-Y quadricepsplasty, and two knees (2.9\%) required an osteotomy of the tibial tubercle. A lateral retinacular release was performed in ten knees (14.5\%) to prevent lateral subluxation of the patella and improve extensor mechanism tracking. 
Pre and post-operative functional outcomes of each treatment of the patella were compared using Student's t test. Setting a definition of "good outcome" as a functional KSS greater than or equal to 60 , we compared the frequencies of each patellar treatment, extensor mechanism release, and each spacer type between patients with good and poor outcomes. Pearson chi-square was used to analyze categorical variables. Statistical significance was defined as a $\mathrm{P}$ value less than 0.05 .

\section{Results:}

The 54 patients that received patellar resurfacing at the time of reimplantation experienced statistically significant improvements in KSS pain score, functional KSS, and patellar score, from 9.2 to $32.5,29.4$ to 52.2 , and 10.9 to 21.6 , respectively ( $\mathrm{P} \mathrm{b} 0.03$ ). Eleven patients that did not have adequate patellar bone to resurface and retained their unresurfaced patellae at time of re-implantation did not experience significant improvements in their functional outcome. Two patients that received trabecular metal patellar augments had minimal improvements in their pain score from 10.0 preoperatively to 25.0 postoperatively (P N 0.05 ), but results were not statistically significant. Similarly, the patient that underwent patellectomy did not have significant improvements in clinical or function outcome. The one patient that received impaction grafting improved significantly in pain and function, reporting a postoperative functional KSS of 100 (Table 1).

When identifying predictors of an outcome of functional KSS 60 or greater, resurfacing the patella and lower BMI were trending towards significance $(\mathrm{p}=0.08$ and $\mathrm{p}=0.06$ respectively). Use of dynamic vs. static spacer, extensor mechanism release, differences in Charlson index, and age did not seem to statistically affect outcomes (Table 2). 


\section{Discussion:}

Revision surgery for PJI in TKA is complicated by bone loss from tissue destruction, debridement of necrotic material at first-stage revision surgery, difficulty with removal of infected implants, and bone loss in the presence of a cement spacer device [10]. Problems affecting the patellofemoral joint have been identified as the most common cause of failure in primary TKA [11], and appropriate management of the patella during revision TKA has similarly been shown to strongly influence the clinical outcome [12,13]. Complications can include soft tissue impingement, avascular necrosis, patellofemoral instability, extensor mechanism ruptures, and patellar fracture [12].

Controversy still exists regarding the efficacy of patellar resurfacing in primary TKA [14-17]. While many authors have addressed the issue of patellar revision in aseptic TKA $[12,18-20]$, this study is the first to our knowledge to specifically address management of the patella in two-stage revision for infected TKA.

The first objective was to determine the success rate of the various treatment options for the patellofemoral joint at reimplantation. The majority of patients, $50(72.5 \%)$ were treated with patellar resurfacing at re-implantation, and this led to significant improvement in postoperative Knee Society Pain and Function Scores, as well as Patellar Scores (P b 0.003). In a study by Patil et al looking at patellar resurfacing in revision TKA, no significant difference in outcome was found between resurfacing and non-resurfacing in aseptic patients. However, a subgroup of patients who had revision for infection noted a significant clinical improvement with patella resurfacing [20]. Patelloplasty has been reported to have outcome inferior to that of resurfacing in primary TKA [21]. While early results of patelloplasty in revision surgery are satisfactory, Knee Society scores show deterioration with time [22]. The eleven patients in this study who 
were treated with patelloplasty did not experience any significant improvement in their outcome measures.

Hanssen reported significant improvement in post-operative Knee Society pain and function scores in a series of nine patients who had bone grafting of a patellar shell performed at the time of revision. The technique involves creation of a retropatellar soft tissue pouch which is filled with bone graft. Patellar thickness was largely maintained at final follow up [19]. Only one patient in our study had this performed, and they obtained significant benefit, reporting a score of post operative functional Knee Society score of 100.

Good outcomes have been reported with porous tantalum augments in the setting of patellar bone deficiency $[23,24]$. To be effective, they require a vascularized bony patellar remnant $[24,25]$. The two patients in our study treated with these augments did not achieve significant improvement in their Knee Society or Feller scores. Similarly, the one patient who had patellectomy performed showed no improvement in their outcome. Patellectomy has historically been reported as showing a poor outcome, with inferior quadriceps strength, easy fatigue and reduced active range of motion post procedure and delayed disruption of the extensor mechanism [23].

The second objective of the study was to identify any prognostic factors which would negatively impact on the fate of the patellofemoral fate following revision arthroplasty for infection. A functional Knee Society Score of less than 60 was taken as implying a poor outcome. Hypothesized predictors for poorer outcome included patellar treatment other than resurfacing, the type of spacer used (dynamic versus static) at first stage revision, whether an extensor release was performed, Charlson index of comorbidities, advanced age and BMI. 
There is evidence to suggest that there may be less bone loss, and better postreimplantation range of motion of the knee with use of an articulating spacer [26,27]. There is little evidence available outlining the effect of extensor release on revision knee arthroplasty outcome, but Barrack reported poorer outcomes in patients who had quadriceps turndown and tibial tubercle osteotomy versus quadriceps snip [28]. Neither of these variables reached statistical significance in predicting a poor outcome. In the same way, advanced age and higher incidence of comorbidities were not prognostic for a poorer outcome in this study.

Patients with increased BMI do not achieve the same improvement in outcome scores post primary TKA $[29,30]$. Although there was a trend towards patients with lower BMI having a better outcome, the difference was not significant. Patients who had patellar resurfacing performed also showed a trend toward having a post-operative KSS score greater than 60, but this was not a significant finding either.

Limitations of the study include the retrospective nature, the relatively small study group (69 knees in 69 patients), the absence of a control group and the short duration of follow up (average of 4.5 years follow up, range one to 10.9 years). Revision procedures for infected total knee arthroplasty are very complex procedures, and the varying condition of the bone and soft tissues do not make for a homogeneous patient population. Furthermore, there was potential for bias on the part of the treating surgeon, although in all cases an attempt was made to resurface the patella where this was deemed feasible. Finally, we compared the demographic data of patients who underwent patellar resurfacing to those who did not. There was no significant difference in age, gender or Charlson comorbidity index between the two groups (data not shown), but BMI was significantly lower in the group who underwent patellar resurfacing than in the group who had another treatment of their patella $(31.6 \pm 8.7$ vs $38.7 \pm 15, \mathrm{P}=0.043)$. This 
might suggest that a lower BMI could influence the improvement in outcome seen with patellar resurfacing, although we did not find that BMI was a significant predictor of good outcome (KSS N 60) with revision TKA. Similarly, in a subgroup analysis of patients undergoing revision TKA, Singh et al noted that increased BMI was not associated with worse pain outcomes [31]. In conclusion, this study shows that patients undergoing revision TKA have a significantly better outcome if patellar resurfacing is performed. Factors postulated to lead to poorer patellofemoral function, including extensor mechanism release and type of spacer used after first stage debridement, did not significantly affect outcome following TKA reimplantation. Salvage procedures for treatment of patellar bone loss, with the exception of patellar bone grafting in one case, provided minimal benefit for the patient. We recommend that every effort should be made to minimize bone loss in first stage resection to allow for resurfacing during reimplantation, as inability to resurface the patella at the time of reimplantation may adversely affect patient outcome. 


\section{References:}

1. Wilson MG, Kelley K, Thornhill TS. Infection as a complication of total knee replacement arthroplasty. Risk factors and treatment in sixty-seven cases. J Bone Joint Surg Am 1990;72(6):878.

2. Windsor RE, Bono JV. Infected total knee replacements. J Am Acad Orthop Surg 1994;2(1):44.

3. Hsu YC, et al. Antibiotic-loaded cement articulating spacer for 2-stage reimplantation in infected total knee arthroplasty: a simple and economic method. J Arthroplasty 2007;22(7):1060.

4. Hsu CS, et al. Two-stage revision of infected total knee arthroplasty using an antibioticimpregnated static cement-spacer. Chang Gung Med J 2008;31(6):583.

5. Garvin KL, Scuderi G, Insall JN. Evolution of the quadriceps snip. Clin Orthop Relat Res 1995(321):131.

6. Della Valle CJ, Berger RA, Rosenberg AG. Surgical exposures in revision total knee arthroplasty. Clin Orthop Relat Res 2006;446:59.

7. Charlson ME, et al. A new method of classifying prognostic comorbidity in longitudinal studies: development and validation. J Chronic Dis 1987;40(5):373.

8. Insall JN, et al. Rationale of the Knee Society clinical rating system. Clin Orthop Relat Res 1989(248):13.

9. Feller JA, Bartlett RJ, Lang DM. Patellar resurfacing versus retention in total knee arthroplasty. J Bone Joint Surg Br 1996;78(2):226.

10. Calton TF, Fehring TK, Griffin WL. Bone loss associated with the use of spacer blocks in infected total knee arthroplasty. Clin Orthop Relat Res 1997(345):148. 
11. Leopold SS, et al. Isolated revision of the patellar component in total knee arthroplasty. $\mathrm{J}$ Bone Joint Surg Am 2003;85-A(1):41.

12. Maheshwari AV, et al. How to address the patella in revision total knee arthroplasty. Knee 2009;16(2):92.

13. Parker DA, Dunbar MJ, Rorabeck CH. Extensor mechanism failure associated with total knee arthroplasty: prevention and management. J Am Acad Orthop Surg 2003;11(4):238.

14. Wood DJ, et al. Patellar resurfacing in total knee arthroplasty: a prospective, randomized trial. J Bone Joint Surg Am 2002;84-A(2):187.

15. Waters TS, Bentley G. Patellar resurfacing in total knee arthroplasty. A prospective, randomized study. J Bone Joint Surg Am 2003;85-A(2):212.

16. Burnett RS, et al. Patellar resurfacing compared with nonresurfacing in total knee arthroplasty. A concise follow-up of a randomized trial. J Bone Joint Surg Am 2009;91(11):2562.

17. Nizard RS, et al. A meta-analysis of patellar replacement in total knee arthroplasty. Clin Orthop Relat Res 2005(432):196.

18. Garcia RM, et al. Management of the deficient patella in revision total knee arthroplasty. Clin Orthop Relat Res 2008;466(11):2790.

19. Hanssen AD. Bone-grafting for severe patellar bone loss during revision knee arthroplasty. J Bone Joint Surg Am 2001;83-A(2):171.

20. Patil N, et al. Patellar management in revision total knee arthroplasty: is patellar resurfacing a better option? J Arthroplasty 25(4):589.

21. Cameron HU. Comparison between patellar resurfacing with an inset plastic button and patelloplasty. Can J Surg 1991;34(1):49. 
22. Parvizi J, et al. Patellar component resection arthroplasty for the severely compromised patella. Clin Orthop Relat Res 2002(397):356.

23. Nasser S, Poggie RA. Revision and salvage patellar arthroplasty using a porous tantalum implant. J Arthroplasty 2004;19(5):562.

24. Nelson CL, et al. Use of a trabecular metal patella for marked patella bone loss during revision total knee arthroplasty. J Arthroplasty 2003;18(7 Suppl 1):37.

25. Ries MD, et al. Porous tantalum patellar augmentation: the importance of residual bone stock. Clin Orthop Relat Res 2006;452:166.

26. Fehring TK, et al. Articulating versus static spacers in revision total knee arthroplasty for sepsis. The Ranawat Award. Clin Orthop Relat Res 2000(380):9.

27. Park SJ, et al. Comparison of static and mobile antibiotic-impregnated cement spacers for the treatment of infected total knee arthroplasty. Int Orthop 2010;34(8):1181.

28. Barrack RL, et al. The Ranawat Award. Comparison of surgical approaches in total knee arthroplasty. Clin Orthop Relat Res 1998(356):16.

29. Yeung E, et al. The effect of obesity on the outcome of hip and knee arthroplasty. Int Orthop. 35(6):929.

30. Dowsey MM, et al. The impact of pre-operative obesity on weight change and outcome in total knee replacement: a prospective study of 529 consecutive patients. J Bone Joint Surg Br. 92(4):513.

31. Singh JA, Gabriel SE, Lewallen DG. Higher body mass index is not associated with worse pain outcomes after primary or revision total knee arthroplasty. J Arthroplasty 26(3):366 e1. 
